\title{
Persistent nocturnal cough: randomised controlled trial of high dose inhaled corticosteroid
}

\author{
M J Davies, P Fuller, A Picciotto, S A McKenzie
}

\begin{abstract}
Objective-To investigate the effect of a short course of inhaled corticosteroid in the treatment of isolated and persistent nocturnal cough in children.

Design-Randomised double blind placebo controlled study.

Setting-Subjects' homes in east London, England.

Subjects-Consecutively referred children, 1-10 years old, with persistent nocturnal cough.

Interventions-Placebo or fluticasone propionate $1 \mathrm{mg}$ twice daily for three nights and $500 \mu \mathrm{g}$ twice daily for 11 nights. Videotaping of children at night: two nights' baseline, nights 3 and 4 after three days of inhaled corticosteroid, and nights 15 and 16.

Main outcome measure-A fall in $75 \%$ of coughs from baseline.

Results-50 subjects were recruited. The median number of coughs in the baseline period for the inhaled corticosteroid group and placebo group were 92 and 71 , respectively $(p=0.43)$ and, on nights 15 and 16,8 and 36 , respectively $(p<0.01)$. Compared to baseline, both groups of subjects improved significantly by nights 15 and $16(p<0.01 ; p<0.01)$. Comparing the inhaled corticosteroid and placebo groups, coughs fell to a median of $22 \%$ and $57 \%$ of baseline totals on nights 3 and 4, respectively $(p=0.38)$, and $8 \%$ and $35 \%$ on nights 15 and 16, respectively $(p=0.02) .17$ of 24 subjects on inhaled corticosteroid who completed the study and 8 of 23 on placebo improved by $75 \%$ after two weeks $(p=0.03)$.
\end{abstract}

Conclusions-Children with persistent nocturnal cough improve in two weeks after referral on placebo. There is a modest benefit from a two week course of high dose inhaled corticosteroid.

(Arch Dis Child 1999;81:38-44)

Keywords: persistent nocturnal cough; cough variant asthma; inhaled corticosteroid

Until recently cough and night time symptoms were not mentioned in the clinical features of childhood asthma. ${ }^{1-3}$ The most recent British asthma guidelines state "criteria for defining asthma in the presence of chronic or recurrent cough have not been adequately defined". ${ }^{4}$ It seems that many children with persistent isolated cough, in the absence of other respiratory symptoms, are now considered asthmatic. ${ }^{5-7}$ However, clinical and epidemiological aspects of isolated persistent cough in childhood differ from childhood asthma ${ }^{59}$ and only the minority of night time coughers have asthma. ${ }^{10}$ In one questionnaire study, ${ }^{5}$ isolated cough was associated with air pollution and dampness in the home, whereas the triad of cough, wheeze, and breathlessness was related to allergic history and preterm birth. In another population study, ${ }^{8}$ children with isolated recurrent cough resembled healthy controls with respect to atopic status, lung function, and response to cold air challenge. In a cross sectional epidemiological study investigating the validity of persistent nocturnal cough as an independent marker of childhood asthma, ${ }^{9}$ the clinical features of children with persistent nocturnal cough resembled those of an asymptomatic group more closely than an asthmatic population.

The term "cough variant asthma" was first used to describe those patients who presented with cough as a single manifestation of their asthma, and who seemed to respond to bronchodilators or short term corticosteroid medication. ${ }^{11}$ Other observations have described this, ${ }^{12-16}$ but until recently there have been no rigorously controlled trials of treatment.

There are several good reasons to consider treating persistent nocturnal cough. Parents complain of their child's and their own loss of sleep and worry about their child choking to death at night. ${ }^{17}{ }^{18}$ In asthmatic children, nocturnal disturbance caused by cough has been related to poor sleep and psychological functioning, ${ }^{19}$ and these benefit from improved management of the asthma. If isolated persistent nocturnal cough is related to asthma, then asthma medication might be expected to help in the same way.

One of the main difficulties of studying cough and its response to intervention is that parents' reports of cough compared to objective recordings of their children's nocturnal symptoms are quantitatively inaccurate. ${ }^{20-22}$ Cough counters are particularly important in assessing the efficacy of antitussive medications. ${ }^{23}$ Tape recorders ${ }^{2022}$ and portable devices ${ }^{24}{ }^{25}$ have been used to count cough. An audiovisual method for recording nocturnal cough has been successfully used in environments not suitable for sound only. ${ }^{26}$ Videorecording allows accurate identification of the source of cough where children are co-sleepers.

There is no proven treatment for children with recurrent or chronic cough not associated with wheeze. Cough linctuses, antibiotics, and now asthma treatments are all used..$^{527}{ }^{28}$ In Kelly's study of subjects in Liverpool between 1991 and 1993 the overall respiratory symptom prevalence including "cough alone" 
seemed to be unchanged, ${ }^{5}$ although prescribing of asthma medication increased. Official recommendations for treatment of recurrent cough view it as an asthma variant. ${ }^{29}{ }^{30}$ The 1993 guidelines of the British Thoracic Society state that for those children under 5 years old "when the major or only symptom is chronic cough, a positive response to a therapeutic trial of $\beta_{2}$ agonist and/or inhaled corticosteroid therapy may help". ${ }^{29}$

In this study we aimed to investigate whether a short course of high dose inhaled corticosteroid is better than placebo in the treatment of isolated persistent nocturnal cough.

\section{Methods}

SUBJECTS

Subjects aged 1-10 years with persistent nocturnal cough were referred by their general practitioners and from ambulatory hospital patients. General practitioners in east London were asked by letter to refer children with persistent nocturnal cough but without other symptoms or signs. Persistent nocturnal cough was defined as reported coughing at night for more than three weeks. Investigations such as chest radiographs were done when clinically indicated. Children with current upper respiratory tract infection, a history of wheeze or fever, and those with an identifiable cause for their cough, such as whooping cough, persistent nasal discharge or large tonsils, were excluded.

\section{STUDY DESIGN}

Parents of those subjects who fitted the study criteria were interviewed using a questionnaire to record demography, family history, and housing conditions. Children were examined by paediatricians. Blood for the estimation of total serum IgE was drawn, and skin prick tests (house dust mite, grass pollens, cat and dog danders, feathers, and positive and negative controls) were undertaken. Wheal sizes $>3 \mathrm{~mm}$ were considered positive. All children at the start of the study had not received treatment with corticosteroids in the month before entry.

To measure nocturnal coughing objectively, a time lapse video recorder (Panasonic Ag6040), and a camera with infra red light (JVC TK-S240E) and a microphone were used to videotape the children in their home environment. ${ }^{26}$ Codings were made using the Observer videotape analysis system for behavioural research (Noldus Information Technology, Tracksys, Nottingham, UK). Individual coughs and cough bouts were counted between the time of being put to bed and rising the following morning. ${ }^{18}$ Bouts were defined as a series of coughs with less than five seconds between each cough.

\section{PILOT STUDY}

In a pilot study consecutive subjects were approached to find out whether families would agree to home studies, to judge the rate of recruitment, to ensure that equipment would not be tampered with, and to enable clear definitions of cough to be coded reliably. Interrater reliability for the codes is $>90 \%{ }^{18}$ It was estimated that recruitment of 30 subjects per year was possible. Because of the variability from night to night - there could be no coughing on one night and 100 coughs the next-it was decided to record nights in pairs. Any more than this would have been impractical. Most of those eligible ( $>50 \%$ ) were preschool children and so it was decided that lung function studies would not be undertaken. We decided that children would be enrolled with a minimum of six cough bouts as this seemed to reflect sufficient coughing to detect change if there was to be any.

\section{CALCULATIONS OF NUMBERS NEEDED}

Calculations were based on the assumption that there would be a $35 \%$ reduction in cough over the 14 days if nothing was given. A difference between treatment and placebo groups of $40 \%$ was considered clinically significant. With $\alpha=0.02$ and $\beta=0.1$ it was calculated that 25 patients would be needed on either side of the study to show this difference, if it existed, with confidence.

PROTOCOL

Following consent given by the parents of children who were currently coughing, recording usually began within the week. Two baseline nights were recorded to measure coughing before beginning treatment. Children with more than six bouts over the two nights were included in the study. Subjects were randomly allocated by our pharmacist to receive either inhaled corticosteroid (fluticasone propionate) or placebo by metered dose inhaler. Parents and children were shown how to use this with a spacer. The study was double blind. Inhalers were weighed before and after the study. The proportion of the expected total dosage taken in two weeks was calculated and this was a measure of apparent adherence to treatment. After three days of treatment with fluticasone at $1 \mathrm{mg}$ twice daily, videorecordings were made during nights 3 and 4 . After a further 11 days of fluticasone $500 \mu \mathrm{g}$ twice daily, videorecordings were made during nights 15 and 16 .

\section{DATA ANALYSES}

Data were analysed using Nanostat (Alphabridge Ltd, London, UK). Demographic data were described using percentages. Correlation coefficients described the relation between individual coughs and cough bouts. Wilcoxon testing was used to compare total baseline coughs with total coughs on nights 3 and 4, and nights 15 and 16 for both the inhaled corticosteroid and placebo groups. Mann-Whitney U tests were used to compare total individual coughs and cough bouts in the inhaled corticosteroid and placebo groups. Chi squared testing was used to compare numbers in each group with $75 \%$ improvement over baseline coughs. For children given inhaled corticosteroid, $\chi^{2}$ testing was used to compare those with one positive skin prick test, and those with $\operatorname{IgE}>100 \mathrm{Ku} / 1$ with $75 \%$ improvement at nights 15 and 16 . T testing was used to compare $\log$ IgE of those on inhaled cortico- 
Table 1 Recruitment and demographic data of subjects referred

\begin{tabular}{llc}
\hline & $\begin{array}{l}\text { Number fitting } \\
\text { entry criteria } \\
(n=131)\end{array}$ & $\begin{array}{l}\text { Total } \\
\text { studied } \\
(n=50)\end{array}$ \\
\hline Sex (female/male) & $77 / 54$ & $30 / 20$ \\
Age 1-4 years & $77(58.8)$ & $29(58)$ \\
Age 5-9 years & $54(41.2)$ & $21(42)$ \\
English not first language & $58(44.3)$ & $28(56)$ \\
Co-sleepers/room share & $82(62.6)$ & $33(66)$ \\
House with damp & $34(26)$ & $11(22)$ \\
House with smoker(s) & $66(50.4)$ & $22(44)$ \\
Pet owners & $26(19.8)$ & $8(16)$ \\
Social class & $11(8.39)$ & $5(10)$ \\
$\quad$ I & $22(16.8)$ & $10(20)$ \\
II & $38(29)$ & $13(26)$ \\
III & $18(13.7)$ & $6(12)$ \\
IV & $7(5.3)$ & $3(6)$ \\
V & $32(24.4)$ & $13(26)$ \\
Unemployed/student & $3(2.3)$ & $0(0)$ \\
Unspecified social class & &
\end{tabular}

Data are $\mathrm{n}(\%)$

Table 2 Duration of cough and medication tried

\begin{tabular}{ll}
\hline & $\begin{array}{l}\text { Main study } \\
\text { subjects } \\
(n=50)\end{array}$ \\
\hline $\begin{array}{l}\text { Reported duration of cough } \\
\quad \text { Median }\end{array}$ & 48 weeks \\
$\quad$ Range & $6-364$ weeks \\
Reported medication tried & \\
$\quad$ Cough medicine (self prescribed) & 43 \\
$\quad$ Antibiotics & 46 \\
$\quad$ Asthma medication & \\
$\quad$ Bronchodilators & 31 \\
$\quad$ Inhaled corticosteroids & 10 \\
Reported exercise limitation because of cough & 22 \\
School days lost due to cough since coughing began \\
$\quad$ Median & 3 \\
Range & $0-90$ \\
\hline
\end{tabular}

Table 3 Treatment adherence

\begin{tabular}{lrc}
\hline Adherence & ICS & Placebo \\
\hline$>70 \%$ contents used & 20 & 15 \\
$50-70 \%$ & 3 & 0 \\
$30-50 \%$ & 1 & 1 \\
$<30 \%$ & 1 & 3 \\
Canisters not returned & 1 & 4 \\
\hline
\end{tabular}

ICS, inhaled corticosteroid.

steroid who improved by $>75 \%$ with those who did not on nights 15 and 16 . Correlation coefficient described the relation between log IgE and percentage change from baseline on nights 15 and 16 .

ETHICS

The study was approved by the East London and City ethics committee. All families were given verbal and written information about the study and they gave written consent, for both videotaping and entering the drug trial at recruitment.

\section{Result}

SUBJECTS AND DEMOGRAPHIC DATA

The demographic data of subjects fitting inclusion criteria and subjects eventually studied are described in table1. On inspection of the data, potential subjects and those studied do not differ in any important way. Exercise limitation, school days lost, previous medication, and length of time coughing are recorded in table 2.

RECRUITMENT AND DROPOUTS

There were 131 children eligible for the study. All but two of 51 children excluded at the time of the first visit were either not coughing at the time of referral (29) or refused to enter the study (20). Of 80 who entered the study, a further 30 were excluded because they did not cough enough, had another illness - for example, whooping cough - or were lost to follow up. Fifty children in total were therefore recruited.

\section{ADHERENCE TO TREATMENT}

Adherence with trial medication is shown in table 3 . Inhalers were not returned by one subject in the inhaled corticosteroid group and four in the placebo group. Twenty of 25 subjects given inhaled corticosteroid and 15 of 20 on placebo were apparently given $>70 \%$ of their trial medication. All subjects who returned inhalers were given some of the medication.

INDIVIDUAL COUGHS AND COUGH BOUTS Numbers of individual coughs and cough bouts were highly correlated (correlation coefficient $=0.95 ; \mathrm{p} \leqslant 0.0001)$.

Twenty six subjects received inhaled corticosteroid and 24 were on placebo. Twenty two subjects in each group had complete recordings on nights 3 and 4, and 24 and 23 subjects respectively had complete recordings on nights 15 and 16. Total coughs for each subject on the baseline nights, on nights 3 and 4, and nights 15 and 16 are shown in fig 1 . The percentage changes over the period of the study for individual coughs are shown in fig 2. The median total numbers of coughs on the baseline nights, nights 3 and 4, and nights 15 and 16 are shown in table 4 . Both groups had significantly fewer coughs on nights 3 and 4, and nights 15 and 16 than during the baseline period $(p<0.01, p<0.01)$. The median percentages of baseline coughs on nights 3 and 4, and nights 15 and 16 are also presented; the difference between the inhaled corticosteroid and placebo groups is significant on nights 15 and 16 but not on nights 3 and 4 . The results for cough bouts showed similar treatment differences. Since bouts correlated closely with individual coughs the results are not presented.

Twelve of 22 subjects on inhaled corticosteroid and 10 of 22 on placebo improved by $75 \%$ on nights 3 and $4\left(\chi^{2}=0.09 ; \mathrm{p}=0.76\right)$ and 17 of 24 and 8 of 23 on nights 15 and 16 $\left(\chi^{2}=4.8 ; \mathrm{p}=0.03\right)$, respectively. Of 20 children with complete studies and who had inhaled corticosteroid, 11 were $75 \%$ better on nights 3 and 4 and all remained better at nights 15 and 16 . For 21 children with complete studies taking placebo, nine were $75 \%$ better on nights 3 and 4 but four of these deteriorated by nights 15 and 16 .

Table 5 shows the changes in skin prick test, IgE, and family history of atopy for both the inhaled corticosteroid and placebo groups. There was no significant relation between any of these variables and $75 \%$ improvement on nights 15 and 16 in the inhaled corticosteroid group. The geometric mean of $\operatorname{IgE}$ in the inhaled corticosteroid group who were $75 \%$ better on nights 15 and 16 was $37.2 \mathrm{Ku} / 1$ and for those who were not it was $14.8 \mathrm{Ku} / 1$ 


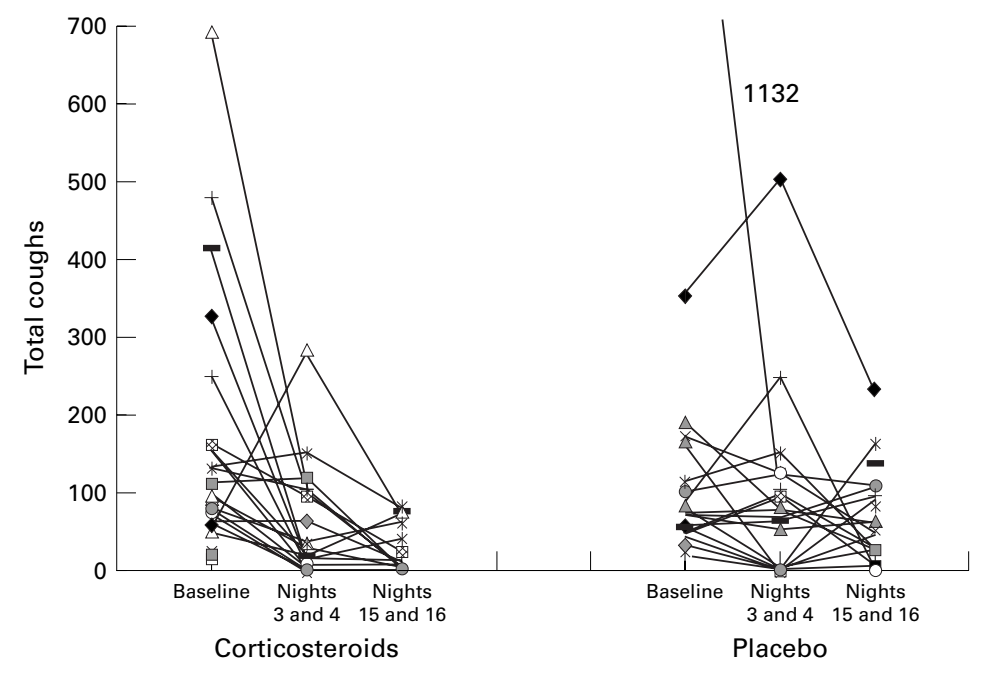

Figure 1 Total cough counts.

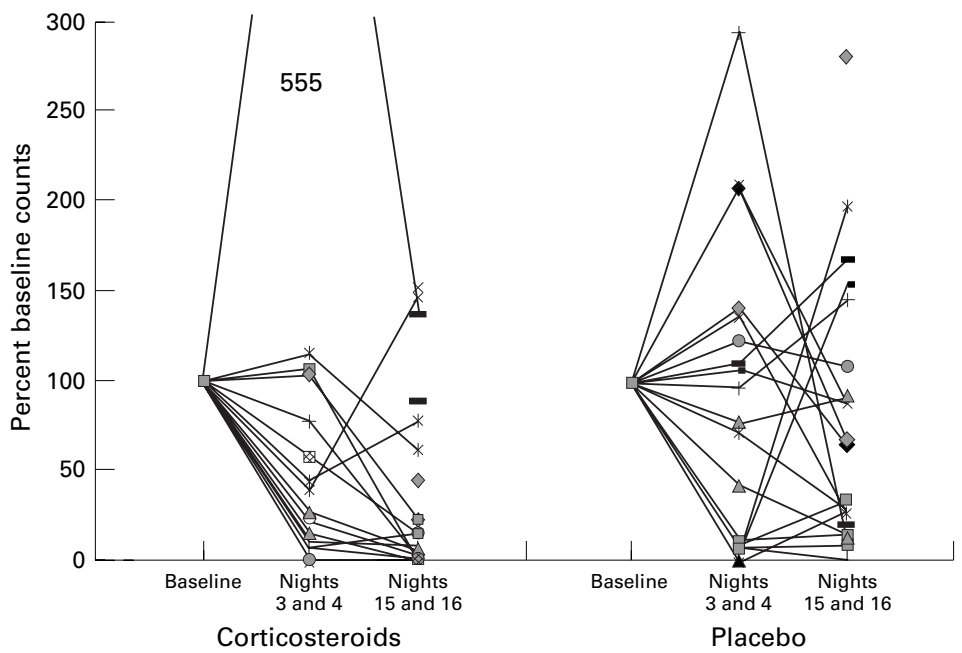

Isolated points on nights $15 \& 16$ represent those where data for nights $3 \& 4$ were not available

Figure 2 Percentage change in cough counts.

Table 4 Cough counts

\begin{tabular}{|c|c|c|c|c|c|c|}
\hline \multirow[b]{2}{*}{ Study nights } & \multicolumn{2}{|c|}{ Total cough counts } & \multirow[b]{2}{*}{ p Value } & \multicolumn{2}{|c|}{$\begin{array}{l}\text { Cough counts } \\
\text { (percentage of } \\
\text { baseline totals) }\end{array}$} & \multirow[b]{2}{*}{ p Value } \\
\hline & $I C S$ & Placebo & & $I C S$ & Placebo & \\
\hline Baseline & $\mathrm{n}=26$ & $\mathrm{n}=24$ & & & & \\
\hline Median & 92 & & 0.43 & & & \\
\hline Interquartile range & $51-153$ & $44-103$ & & & & \\
\hline Nights 3 and 4 & $\mathrm{n}=22$ & $\mathrm{n}=22$ & & & & \\
\hline Median & 23 & 66 & 0.80 & 22 & 57 & 0.38 \\
\hline Interquartile range & $4-100$ & $2-102$ & & $3-73$ & $8-120$ & \\
\hline Nights 15 and 16 & $\mathrm{n}=24$ & $\mathrm{n}=23$ & & & & \\
\hline Median & 8 & 36 & $<0.01$ & 7 & 32 & 0.02 \\
\hline Interquartile range & $1-26$ & $11-90$ & & $1-49$ & $15-92$ & \\
\hline
\end{tabular}

ICS, inhaled corticosteroid.

Table 5 Atopic markers of subjects who completed nights 15 and 16

\begin{tabular}{llllll}
\hline & \multicolumn{2}{l}{ Inhaled corticosteroids } & & \multicolumn{2}{l}{ Placebo } \\
\cline { 2 - 3 } \cline { 6 - 7 } Markers & $>75 \%$ better & $<75 \%$ better & & $>75 \%$ better & $<75 \%$ better \\
\hline Positive skin prick test $(\mathrm{n}=45)$ & $6 / 16(38)$ & $1 / 7(14)$ & & $1 / 7(14)$ & $3 / 18(20)$ \\
IgE $>100 \mathrm{Ku} / 1(\mathrm{n}=42)$ & $4 / 14(29)$ & $1 / 6(17)$ & & $0 / 8(0)$ & $3 / 14(21)$ \\
Positive family history $(\mathrm{n}=47)$ & $14 / 17(82)$ & $6 / 7(86)$ & & $7 / 8(88)$ & $11 / 18(75)$ \\
\hline
\end{tabular}

Values are $\mathrm{n}(\%)$. $(t=1.14 ; \mathrm{p}=0.27)$. For all patients on inhaled corticosteroid $\log \operatorname{IgE}$ did not correlate with percentage improvement on nights 15 and 16 (correlation coefficient $0.08 ; \mathrm{p}=0.73$ ).

FOLLOW UP

Twenty seven children were followed up either as outpatients or on the telephone at six months to a year following the study. Of those contacted, the parents of 18 said their child's cough was better or completely gone, four reported no change, one had had hospital admissions with "chest infections", and four were on regular asthma medication. Seventeen lost contact with the study, despite repeated telephone calls and letters. Of the last six who completed the study less than six months ago, four are better, one is still coughing, and one has been prescribed a further course of inhaled corticosteroid.

\section{Discussion}

We undertook this study following the British Thoracic Society's guidelines recommending a therapeutic trial of inhaled corticosteroid for chronic cough ${ }^{29}$ and following a survey of our local family doctors' prescribing habits for recurrent and persistent cough, ${ }^{7}$ which suggested that treatment of persistent nocturnal cough with inhaled corticosteroid was considered by many. Parents seem keen to have medication for their children's persistent nocturnal cough.

The demographic data of children recruited resembled those eligible but not recruited. We had approached local general practitioners asking them to refer subjects for the study to try to reflect as far as possible children with cough in the community. We acknowledge that perhaps those with the most troublesome cough or most worried parents may have been referred.

There were more girls than boys, which is unlike the sex ratio of children with asthma and coughers in other studies. ${ }^{931}$ Our numbers are small, however. Others have shown that preschool children are those whose parents most often report nocturnal cough. ${ }^{32}$ We believe that in selecting consecutive referrals willing to enter the study we have recruited a sample which reflects the population. Others have selected only older children. ${ }^{33}$ The social class status of our subjects ${ }^{34}$ and the proportion of subjects who live with parents who smoke in this study (44\%) are not dissimilar from that of the local population. ${ }^{35}$ The number who say they live in damp houses (22\%) is much higher than the national average $(7 \%),{ }^{36}$ and the number who share a room or co-sleep reflects local overcrowding. ${ }^{36}$

The median duration of the cough was almost a year. The results of our study of the children given placebo suggest that even in a two week period cough is very variable. Nevertheless this continued cough could be perceived by parents to be persistent. We have shown that parents correctly know when their child is coughing ${ }^{18}$ although they cannot tell how much. ${ }^{22}$ Most children had been given antibiotics and some asthma treatment includ- 
ing inhaled corticosteroid. This is in accordance with the prescribing intentions of our local general practitioners. ${ }^{7}$ Many had exercise limitation because of the cough. It is impossible to know whether this is because of related shortness of breath caused by unperceived wheeze or just because of the difficulty of exercising while coughing. School or nursery absence because of cough was very variable but was common enough to be of concern.

By using videorecordings, ${ }^{26}$ we have been able to make observations of children with nocturnal cough in their own homes. This method eliminates difficulties with diary card reporting and the problems associated with recording cough of co-sleepers. Others have used different methods ${ }^{24}{ }^{25}$ which have recorded coughs over a 24 hour period. Most parents report that it is nocturnal cough which is more problematic than cough during the day. ${ }^{18}$ Our software allowed us to measure both individual coughs and cough bouts. This was important because children could potentially have only one cough bout made up of say 20 individual coughs. Conversely, nocturnal cough could occur as a single individual cough per bout, but the child may have 20 bouts during the night. Individual coughs and cough bouts were very closely correlated, however.

Many eligible subjects did not cough on the baseline nights. Over $25 \%$ of subjects on placebo had fewer than six bouts on nights 15 or 16 , and so it is not surprising that between the time of recruitment and recording some potential recruits stopped coughing.

One or more positive skin prick tests were shown in $24 \%$ of our subjects, a similar percentage to that in Clifford's study of coughers aged 7-11 years. ${ }^{37}$ In a study of preschool children who coughed and controls of the same age, $63 \%$ and $10 \%$, respectively, were atopic by the same definition. ${ }^{31}$ It seems that the atopic status of children who cough is intermediate between asymptomatic children and children who wheeze. ${ }^{8} 313738$ A family history of atopy in first degree relatives was $64 \%$, much higher than has been found in other studies. ${ }^{891531} \mathrm{We}$ cannot explain this, other than to suggest that, because we had a large number of subjects whose parents' first language was not English, many parents perhaps understood something different by the terms asthma, eczema, and hay fever. We have examined our small number of subjects for a relation between markers of atopic status and improvement in coughing in the group given inhaled corticosteroid. No strong relation was shown.

As we expected from our pilot study that over $50 \%$ of our subjects would be aged $1-5$ years, we did not include lung function testing as part of the protocol. With the development of the measurement of airways resistance by the interrupter technique, ${ }^{39}$ and the measuring of response to bronchodilators, preschool children with respiratory symptoms can now have some assessment of respiratory function.

We chose a very high dose of inhaled corticosteroid for three days to imitate a three day course of prednisolone used in asthma, when a benefit of treatment over placebo would

\section{Key messages}

- It is sometimes believed that children with recurrent or persistent cough have asthma and are prescribed asthma medication

- This study of a group of children with cough has shown that the cough gets better over two weeks and that inhaled corticosteroid, even in very high dosages, offers very little benefit

be expected. ${ }^{40}$ In this study, coughers like asthmatic patients improve over three days even with placebo, but unlike asthmatic patients inhaled corticosteroid conferred no additional benefit. Others have used high doses of budesonide in children hoping for a rapid remission. ${ }^{41}$ Although there was no difference between inhaled corticosteroid and placebo in the improvement in cough after three days, after 14 days the improvement in the inhaled corticosteroid group was greater than in the placebo group, although even the latter group was much better than at the start. The effect in older children with asthma treated with inhaled corticosteroid can be seen at 14 days. ${ }^{42}$ Those on inhaled corticosteroid who had $75 \%$ improvement at three days sustained this at the end of the study. It would be interesting to know if improvement was sustained beyond stopping after three days' treatment. Two other studies have examined the effect of corticosteroids on cough and found no benefit. ${ }^{33} 43$ The high dose of corticosteroid we chose could explain the difference in our study. In one study the compliance with taking the corticosteroid was $55 \%$, ${ }^{33}$ which may have had a bearing on the results. In comparison our study was much shorter, probably encouraging better adherence to treatment. The improvement in our placebo subjects- $57 \%$ in the first week and $35 \%$ at the end of the second week - is not dissimilar to that in Chang's placebo subjects of $34 \%$ in $5-7$ days. $^{33}$

Some children with persistent nocturnal cough no doubt have unrecognised wheeze, either because it is poorly perceived by their parents or it is not present at the time they present to a physician. It has also been suggested that this group has a higher wheeze threshold. ${ }^{16}$ The fact that inhaled corticosteroid has helped the coughers in this study does not mean that they necessarily have asthma. Measurements of bronchodilator responsiveness in our laboratory have identified some children with persistent cough who respond in the same way as children who have wheeze. These children could represent children with "cough variant" asthma. Studies of the effect of inhaled corticosteroid on reversibility to bronchodilators in such children should help clarify this. It may be that children with recurrent, persistent, and isolated cough occupy an intermediate clinical position between children with no cough and those with wheeze with respect to atopy, ${ }^{31}$ prognosis for asthma, ${ }^{810}$ family history of asthma, ${ }^{9}$ response 
to bronchodilator, ${ }^{44}$ and episodes of respiratory morbidity. ${ }^{27}$ However, coughers appear to have normal lung function measured by spirometry and airway responsiveness. ${ }^{83}$

In this study, the group given inhaled corticosteroid benefited as a whole from two weeks' treatment. Many of these subjects would have improved in any event. A criticism of clinical trials is that they do not identify the characteristics of those individuals within the group who are likely to benefit from the treatment. This trial is no different. In the small number of subjects tested, atopic status did not point to those likely to be helped. A positive relation between isolated cough and atopic family history as markers for the later development of wheeze has been noted, ${ }^{10}$ and others also found that children with cough and no wheeze had more atopy than controls but less than asthmatics. ${ }^{81}$ Eczema and hay fever, however, occurred no more in coughers than controls. ${ }^{9}$

We suggest that inhaled corticosteroids are not prescribed at the time of presentation of persistent nocturnal cough. The most important observation made in this study is that children improved on placebo. Of those we were able to follow up, most remained better. Since follow up was six months to one year after the study, this could simply reflect the natural history of the tendency of cough to improve with time. ${ }^{45}$ Many parents are able to detect change for the better or worse in their child's coughing. ${ }^{18}$ If inhaled corticosteroids are to be prescribed then a two week high dose course may benefit some of these children. The doses used in this study should not cause irreversible side effects over a two week period but these dosages will have caused cortisol suppression. ${ }^{46}$ Prolonged prescribing at this dosage could have side effects on growth and is certainly not warranted. ${ }^{47}$ There is nothing at present to suggest that treatment with inhaled corticosteroids at the usual doses will be beneficial to children with persistent nocturnal cough. Since we have shown benefit from a very high dose of an inhaled corticosteroid (fluticasone propionate) we are encouraged to undertake a larger study using conventional doses of inhaled corticosteroid to investigate whether a group of children with persistent nocturnal cough who have never wheezed, who are atopic, and who have reversibility of airways resistance or spirometric indices to bronchodilator, are likely to benefit.

We are grateful to the families who took part in this study, to the general practitioners of east London and hospital physicians who kindly referred subjects to us, and to Ms V Bradnam, principal pharmacist. We are especially grateful to GlaxoWellcome for support.

1 Dawson B, Horobin G, Illsley R, Mitchell RA. A survey of childhood asthma in Aberdeen. Lancet 1969;i:827-30.

2 Williams H, McNicol KN. Prevalence, natural history and relationship of wheezy bronchitis and asthma in children: an epidemiological study. $B M \mathcal{F} 1969$;iv:321-5.

3 Phelan P. Asthma in children: epidemiology. BMF 1994;308: 1584-5.

4 British Thoracic Society, National Asthma Campaign, Royal College of Physicians of London, et al. British guidelines on asthma management: review and position statelines on asthma management: revi
ment. Thorax 1997;52(suppl 1).
5 Kelly YJ, Brabin BJ, Milligan PJM, Reid JA, Heaf DH, PearKelly YJ, Brabin BJ, Milligan PJM, Reid JA, Heaf DH, Pear-
son MG. The clinical significance of cough in the diagnosis son MG. The clinical significance of cough in the diagnosis
of asthma in the community. Arch Dis Child 1996;75:489of as.

6 McKenzie SA. Cough-but is it asthma? Arch Dis Child 1994;70:1-2.

7 Picciotto A, Sturdy P, Hubbard M, Naish J, McKenzie SA. Prescribing for persistent cough in children. Respir Med 1998;92:638-41.

8 Wright AL, Holberg CJ, Morgan WJ, Taussig LM, Halonen M, Martinez FD. Recurrent cough in childhood and its 1259-65.

9 Ninan TK, Macdonald L, Russell G. Persistent nocturnal cough in childhood: a population based study. Arch Dis Child 1995;73:403-7.

10 Powell CVE, Primhak RA. Stability of respiratory symptoms in unlabelled wheezy illness and nocturnal cough. Arch Dis Child 1996;75:1-7.

11 Glauster FL. Variant asthma. Ann Allergy 1972;30:457-9.

12 Corrao WM, Marman SS, Irwin RS. Chronic cough as the sole presenting complaint of bronchial asthma. N Engl $\mathcal{F}$ Med 1979;330:633-7.

13 Hannaway PJ, Hopper DK. Cough variant asthma in children. FAMA 1982;247:206-8.

14 Pender ES, Pollack CV. Cough variant asthma in children and adults: case reports and review. F Emerg Med 1990;8:727-31.

15 Spelman R. Two-year follow-up of the management of chronic or recurrent cough in children according to an asthma protocol. Br f Gen Pract 1991;41:406-9.

16 Koh YY, Chae SA, Min KU. Cough variant asthma is associated with a higher wheezing threshold than classic asthma. Clinical and Experimental Allergy 1993;23:696-701.

17 Cornford CS, Morgan M, Ridsdale L. Why do mothers consult when their children cough? Family Practice consult when

18 Fuller P, Picciotto A, Davies M, McKenzie SA. Cough and sleep in inner city children. Eur Respir f 1998;12:426-31.

19 Stores G, Ellis AJ, Wiggs L, Crawford C, Thomson A. Sleep and psychological disturbance in nocturnal asthma. Arch Dis Child 1998;78:413-19.

20 Archer LNJ, Simpson H. Night cough counts and diary card scores in asthma. Arch Dis Child 1985;60:473-4.

21 Hoskyns EW, Thomson A, Decker E, Hutchins A, Simpson $H$. Effect of controlled release salbutamol on nocturnal cough in asthma. Arch Dis Child 1991;66:1209-12.

22 Falconer A, Oldman C, Helms P. Poor agreement between reported and recorded nocturnal cough in asthma. Pediatr Pulmonol 1993;15:209-11.

23 Pirila P, Sovijarvi ARA. Objective assessment of cough. Eur Respir F 1995;8:1949-56.

24 Munyard P, Bush A. How much coughing is normal? Arch Dis Child 1996;74:531-4.

25 Chang AB, Newman RG, Phelan PD, Robertson CF. A new use for an old Holter monitor: an ambulatory cough meter. Eur Respir f 1997;10:1637-9.

26 Picciotto A, Fuller P, Hubbard M, McKenzie SA. Videorecording at night in children's homes. International fournal of Social Research 1998;1:153-61.

27 Levy M, Bell L. General practice audit of asthma in childhood. BMF 1984;289:1 115-18.

28 Kourdoulos D, Schattner P. A survey on the diagnosis and management of asthma in young children. Aust Fam Physician 1997;26(suppl 2):S71-5.

29 British Thoracic Society, British Paediatric Association, Royal College of Physicians of London, King's Fund Centre, National Asthma Campaign, et al. Guidelines on the tre, National Asthma Campaign, et al. Guidelines on

30 Warner JO, Naspitz CK. Third international pediatric consensus statement on the management of childhood asthma. sensus statement on the manag
Pediatr Pulmonol 1998;25:1-17.

31 Lewis HM, Haeney M, Jeacock J, Thomas H. Chronic cough in a hospital population; its relationship to atopy an defects in host defence. Arch Dis Child 1989;64:1593-8.

32 Toop LJ, Howie JGR, Paxton FM. Night cough and general practice research. F R Coll Gen Pract 1986;36:74-7.

33 Chang AB, Phelan PD, Carlin JB, Sawyer SM, Robertson CF. A randomised, placebo controlled trial of inhaled salbutamol and beclomethasone for recurrent cough. Arch Dis Child 1998;79:6-11.

34 Census Manual. East London and City Health Authority, 1991.

35 East London and the City: Health in the east. Annual Public Health Report, 1995-96.

36 Hyndman SJ. Housing dampness and health amongst British Bengalis in east London. Soc Sci Med 1990;30:131-41

37 Clifford RD, Radford M, Howell JB. Prevalence of atopy and range of bronchial response to methacholine in 7 and 11 year old schoolchildren. Arch Dis Child 1989;64:1126-32.

38 Clough JB, Holgate ST. Episodes of respiratory morbidity in children with cough and wheeze. Am $\mathcal{F}$ Crit Care Med 1994; 150:48-53.

39 Phagoo SB, Wilson NM, Silverman M. Evaluation of a new interrupter device for measuring bronchial responsiveness and the response to bronchodilator in 3 year old children. Eur Respir $\mathcal{f}$ 1996;9:1374-80.

40 Deshpande A, McKenzie SA. A short course of steroids for the home treatment of children with acute asthma. $B M \mathcal{F}$ 1986;293:169-70.

41 Volovitz B, Soferman R, Blau H, Nussinovitch M, Varsano I. Rapid induction of clinical response with a short-term high-dose starting schedule of budesonide nebulizing suspension in young children with recurrent wheezing episodes. F Allergy Clin Immunol 1998;101:464-9. 
42 Pedersen S, Hansen OR. Budesonide treatment of moderate and severe asthma in children: a dose response study. $\mathscr{F}$ allergy Clin Immunol 1995;90:29-33.

43 Evald T, Munch EP, Kok-Jensen A. Chronic non-asthmatic cough is not affected by inhaled beclomethasone dipropionate. Allergy 1989;44:510-14

44 Konig P. Cough variant asthma. F Asthma 1991;28:83-4.

45 Brooke AM, Lambert PC, Burton PR, Clarke C, Luyt DK, Simpson $\mathrm{H}$. The natural history of respiratory symptoms in pre-school children. Am 7 Respir Crit Care Med 1995;152: $1872-8$.

46 Agertoft L, Pedersen S. Short-term knemometry and urine cortisol excretion in children treated with fluticasone propionate and budesonide: a dose response study. Eur Respir f 1997;10:1507-12.

47 Todd K, Dunlop J, McNaboe MF, et al. Growth and adrenal suppression in asthmatic children treated with high-dose fluticasone proprionate Lancet 1996;348:27-9.

\section{Inclusion benefit}

The results of clinical trials may not be reflected in ordinary clinical practice. Strict regulation of the conditions under which trials occur may mean that both intervention and control groups are cared for in ways that are different from those that apply outside such trials. Standards of practice may be different in units that undertake clinical trials and may differ between trial and non-trial groups in the same units.

At McMaster University in Hamilton, Canada a trial of antithrombin treatment in premature infants with respiratory distress syndrome (Barbara Schmidt and colleagues. American fournal of Respiratory and Critical Care Medicine 1998;158:470-6) showed an adverse effect of antithrombin. A total of 198 babies was eligible for entry into the trial but 76 were not randomised because parental consent was withheld (38), because consent was not asked for, usually for administrative reasons (24), or because the trial was closed temporarily because of production problems with the antithrombin (14). These eligible, non-randomised babies did worse than those randomised to placebo (Barbara Schmidt and colleagues. Fournal of Pediatrics 1999;134:151-5; see also editorial, Ibid: 130-1). The median duration of mechanical ventilation was 7.1 days in the antithrombin group, 4.8 days in the placebo group, and 6.2 days in the eligible, non-randomised group. Median duration of supplemental oxygen administration was 7.9 days, 5.5 days, and 4.9 days, respectively. Intraventricular haemorrhage was somewhat more frequent in the eligible, non-randomised group compared with the placebo group $(32 \% v 22 \%, \mathrm{p}=0.22)$. The authors argue that the differences seen might not simply be a placebo effect as ordinarily understood.

If patients allocated to placebo do better than those not randomised there are several implications. Placebo groups are likely to be treated according to strict protocol and therefore the finding might be seen as providing justification for protocol or guidelines based practice. Second, the true benefit of the treatment being investigated might be greater than that shown by the difference between treatment and placebo groups (an editorial writer suggests that a less rigorous standard of statistical significance might be acceptable). Third, participation in clinical trials might be regarded as beneficial in itself and the need for ethical standards to protect trial patients might be seen as less compelling. (The same editorialist rejects this proposition on the grounds that patients in trials need protecting not because trials are inherently dangerous but because they alter the doctor-patient relationship.)

The changes brought about by setting up a clinical trial may be complex. They need to be taken into consideration in assessing trial results and the implications for clinical practice in general.

ARCHIVIST 